\title{
Effects of Anticoagulants on Experimental Models of Established Chronic Liver Diseases: A Systematic Review and Meta-Analysis
}

\author{
Rui Zhang $\mathbb{D}^{1,2,3}$ Xiaoquan Huang $\mathbb{D}^{1,2,3}$ Yingyi Jiang $\mathbb{D}^{1,2,3}$ Jian Wang $\mathbb{D}^{1,3}$ \\ and Shiyao Chen $\mathbb{D}^{1,2,3,4}$ \\ ${ }^{1}$ Department of Gastroenterology and Hepatology, Zhongshan Hospital, Fudan University, Shanghai, China \\ ${ }^{2}$ Center of Evidence-based Medicine, Fudan University, Shanghai, China \\ ${ }^{3}$ Shanghai Institute of Liver Disease, Shanghai, China \\ ${ }^{4}$ Endoscopy Center and Endoscopy Research Institute, Zhongshan Hospital, Fudan University, Shanghai, China
}

Correspondence should be addressed to Shiyao Chen; chen.shiyao@zs-hospital.sh.cn

Received 14 August 2020; Revised 9 November 2020; Accepted 28 November 2020; Published 12 December 2020

Academic Editor: Alessandro Granito

Copyright (c) 2020 Rui Zhang et al. This is an open access article distributed under the Creative Commons Attribution License, which permits unrestricted use, distribution, and reproduction in any medium, provided the original work is properly cited.

Objective. The role of anticoagulants in chronic liver diseases is inconclusive. A meta-analysis was thus undertaken to evaluate treatment-related survival and antifibrotic effects in animal models of chronic liver diseases. Methods. A systematic search of the literature took place (up to November 2020), screening for preclinical studies that evaluated anticoagulant effects in animal models of chronic liver diseases. We assessed the quality of methods and the certainty of evidence. Data on outcomes were extracted and pooled into random-effects models. Results. Sixteen studies proved eligible, each assessing anticoagulant use in animals with chronic liver diseases. Generally, the pooled evidence demonstrated that the administration of anticoagulants is preventive against fibrogenesis, as indicated by METAVIR fibrosis scores (risk ratio $=0.66,95 \%$ confidence interval: 0.47 to 0.94 ), portal pressure determinations (mean difference $=-1.39,95 \%$ confidence interval: -2.33 to -0.44 ), inflammatory activity (mean difference $=-169.69,95 \%$ confidence interval: -257.64 to -81.74 ), and indices of hepatic injury, specifically alanine aminotransferase (mean difference $=-82.7,95 \%$ confidence interval: -107.36 to -58.04 ), aspartate aminotransferase (mean difference $=-186.12$, 95\% confidence interval: -254.90 to -117.33 ), albumin (mean difference $=0.59,95 \%$ confidence interval: 0.16 to 1.01 ), and total bilirubin (mean difference $=-0.96,95 \%$ confidence interval: -1.46 to -0.46 ), and it had no impact on animal survival (risk ratio $=1.03,95 \%$ confidence interval: 0.94 to 1.13). Conclusions. Our assessments indicate that in animal models of chronic liver diseases, anticoagulants may alleviate the degree of fibrosis evaluated by the METAVIR score system, portal pressure, inflammatory activity, and serum indices of hepatocellular injury, without impacting survival. High-quality experimental studies are still required.

\section{Introduction}

The liver plays a crucial role in the global hemostatic process through its synthesis and regulation of most pro- and anticoagulant (AC) factors [1]. However, hemostasis may be profoundly disrupted as pathological changes develop in the liver. Fibrosis or cirrhosis shares the final response of the liver to a variety of offending stimuli, as well as a key driver of the natural course of all types of advanced liver disease. Although antifibrotic treatment is a research and a clinical priority, there are presently no drugs licensed antifibrotic drugs for use in humans. Patients with either cirrhosis or NAFLD often are at increased risk of prothrombotic conditions $[2,3]$, as exemplified by the presence of thrombotic occlusion of the portal vein and microthrombotic occlusion of intrahepatic veins and sinusoids in cirrhotic livers, and thrombotic events including portal vein thrombosis (PVT) in NAFLD and its evolutive forms [4, 5]. In addition, thrombosis of intrahepatic vessels is frequently followed by hepatic inflammatory injury, which may eventually aggravate the progression of fibrosis, and leading to worsening of PVT and portal hypertension (PP) [6, 7]. Mechanistically, 
the activated hepatic stellate cells (HSCs) are the key pathogenic initiators of hepatic fibrogenesis. They can be activated by thrombin and FXa and inhibited by anticoagulants to prevent or reduce fibrogenesis [5]. These notions support the potential association between coagulation and fibrosis, and the rationale for the use of anticoagulants that rebalance coagulative parameters as an antifibrotic agent and, ultimately, to prevent PVT and PP.

Anticoagulant therapies, such as heparin (standard or low molecular weight formulations), vitamin $\mathrm{K}$ antagonists, antiplatelet agents, and novel direct oral ACs (NOACs), are widely available for clinical use to interpret the therapeutic effects of ACs in the context of chronic liver diseases. However, the results also remain inconclusive. Chung et al. reported that warfarin administration significantly enhanced therapeutic response rates in patients with cirrhosis and nontumor-related portal vein thrombosis (PVT), compared with controls [8]. Similarly, one RCT also showed that prophylactic administration of enoxaparin for a year improved survival, and prevented PVT development and hepatic decompensation in patients with advanced compensated cirrhosis [9]. On the contrary, Chen et al. failed to demonstrate any benefit to warfarin use in patients with liver cirrhosis [10]. A previously conducted systematic review and meta-analysis of clinical trials addressed the safety and efficacy of AC treatment in patients with cirrhosis and PVT, but the analysis mainly focused on thrombotic recanalization and hepatic complications [11].

In experimental animal models, anticoagulant therapies have also recently emerged as attractive choices to manage a variety of hepatic injuries inflicted by western diets, bile duct ligation (BDL), and carbon tetrachloride $\left(\mathrm{CCl}_{4}\right)$ or thioacetamide (TAA) toxicity $[12,13]$. In addition to their antithrombotic properties, ACs confer antifibrotic and antiinflammatory effects $[14,15]$. The underlying cellular and molecular mechanisms appear to involve activities of hepatic stellate cells (HSCs), endothelial dysfunction, the factor Xa or thrombin, transforming growth factor-beta (TGF$\beta) / S M A D$ signaling, extracellular signal-regulated kinase (ERK) signaling, signal transduction from AKT to c-RAF, AKT signaling, and the nitric oxide (NO) pathway $[13,16-20]$. To date, animal models addressing the effects of ACs on chronic liver diseases have also produced inconclusive results. Cerini et al. showed that enoxaparin reduced liver fibrosis and PP in rats with cirrhosis [21]. Conversely, in models of rats with advanced cirrhosis, enoxaparin did not ameliorate liver function, liver fibrosis, or PP [22]. More importantly, no data on animal models involving chronic liver injuries and anticoagulation have been systematically summarized and reported to date.

To clarify this issue, we comprehensively merged existing preclinical evidence to ascertain the effects of anticoagulant therapies on survival, hepatic fibrosis, PP, hepatic injury, and inflammatory response in animals with established and variably induced chronic liver disease.

\section{Methods}

2.1. Literature Search and Study Selection. This study adhered to the Preferred Reporting Items for Systematic Reviews and
Meta-Analyses (PRISMA) guidelines (Table S1) [23]. Trials selected for review were retrieved through comprehensive searches of specific electronic databases (PubMed, Cochrane Library, and Web of Science) and were limited to articles published between January 2000 and November 2020. The following filters were applied: (rats OR mice OR animal OR experiment OR preclinical) AND ("liver cirrhosis" OR "liver fibrosis" OR "hepatic cirrhosis" OR "liver fibrosis" OR cirrho*) AND (anticoagulant OR aspirin OR enoxaparin OR rivaroxaban). Preclinical, controlled comparative studies of animal disease models with chronic liver disease culminating in hepatic fibrosis/cirrhosis and involving anticoagulant therapy (versus no intervention) were targeted. Grounds for exclusion were as follows: (1) nonanimal or nonoriginal studies, (2) noninterventional studies, (3) experimental animals with chronic liver disease and concurrent hepatocellular carcinoma or bowel inflammation, and (4) models of complications secondary to cirrhosis, without underlying chronic liver disease. Two independent reviewers screened eligible publications, first by title and abstract and then by full text. Disagreements were resolved through discussion with or arbitration by a third reviewer until reaching a consensus.

2.2. Data Extraction. For each study, data on trial execution (first author, year of publication, country of origin, and study design), baseline animal characteristics (animal number, age, species/strain, weight, and sex), disease model (modeling method), type of regimen (anticoagulation or control, dose, and route), and outcome assessments (primary outcomes: survival, degree of fibrosis, PP; secondary outcomes: parameters of hepatic damage and inflammatory response) were collected by predesigned electronic form. METAVIR fibrosis scores [24] served to gauge hepatic fibrosis, restricting animals to stages F2-F4 for this meta-analysis. Collagen deposition was assessed by Sirius red stain. Numbers extracted or recalculated for meta-analysis included mean, standard deviation (SD), standard error of the mean (SEM), and median values, as well as interquartile range. Descriptive analysis was undertaken if data were not extracted.

2.3. Methodological Quality and Risk of Bias Assessments. Two independent reviewers evaluated the quality of data generated by interventional animal studies using the SYRCLE Risk of Bias tool (Systematic Review Center for Laboratory Animal Experimentation, Nijmegen, The Netherlands) [25]. Discrepancies were rechecked by a third person. The checklists entailed the following points: (1) allocation of sequences (adequately generated and applied?), (2) status of study groups (similar at baseline or adjusted for confounders in the analysis?), (3) allocation process (adequately concealed?), (4) animal housing (random throughout the experiment?), (5) caregivers and/or investigators (blinded to each animal intervention during the experiment?), (6) outcome assessments (animals selected at random?), (7) assessor of outcomes (blinded?), (8) incomplete outcome data (adequately addressed?), and (9) study reports (free of selective outcome reporting?). Because many items were reported as 
"unclear," we included four other sources of bias pertaining to study randomization at outcome assessment (item 10), randomization at allocation level (item 11), state funding (item 12), and conflicts of interest (item 13). In items 1-9, "Yes" responses indicated a low risk of bias, whereas "No" indicated high risk, and "Unclear" indicated uncertain risk. In items 10-12, "Yes" indicated reported, and "No or unclear" indicated unreported. For item 13, "Yes" indicated a stated conflict of interest, "No" indicated denial, and "Unclear" indicated no mention.

2.4. Certainty of Evidence Assessment. The Grading of Recommendations Assessment, Development, and Evaluation (GRADE) method was used to evaluate the quality of evidence for each outcome [26]. Four key components were considered, including methodological limitations, relevance, coherence, and adequacy. Regarding the quality of evidence, we firstly presumed that studies were randomized by design and then pursued degrading evaluations. Grades of evidence were designated as very low quality, low quality, moderate quality, or high quality.

2.5. Statistical Analyses. For outcomes other than survival and fibrosis, data were reported as the mean $\pm \mathrm{SD}$, converting any data expressed otherwise (i.e., SEM or median and interquartile range) accordingly [27]. Continuous and dichotomous variables were pooled into a generic inversevariance and random-effects model using Review Manager 5.3 software (Cochrane Collaboration, Copenhagen, Denmark). Statistical heterogeneity $\left(I^{2}\right)$ values assessed across all studies investigated were ranked as follows: $<25 \%$, very low; 25-50\%, low; 50-75\%, moderate; and $>75 \%$, high. Subgroup analyses of primary outcome measures were carried out to identify sources of heterogeneity. Statistical significance was set at $p \leq 0.05$.

\section{Results}

3.1. Literature Selection and Study Characteristics. Once deduplication and preliminary screening were complete, a total of 16 publications met our eligibility criteria (Figure 1). Study characteristics are summarized in Table 1 and Table S2. Twelve of these 16 studies were candidates for meta-analysis. The other four presented nonextractable data reserved for descriptive analysis. Cirrhosis, hepatic fibrosis, NAFLD, and cholestatic liver injury were modeled in four, nine, two, and one of the 16 studies, respectively. The most common agents used to induce hepatic fibrosis/cirrhosis were $\mathrm{CCl}_{4}(75 \%)$ and TAA $(31.25 \%)$. Other modeling strategies included BDL; western diet; choline-deficient, L-amino acid-defined (CDAA) diet; high-fat, high-calorie ( $\mathrm{HF} / \mathrm{HC}$ ) diet; porcine serum; and 1\% dimethylnitrosamine (DMN). Overall, 75\% of animals were male, and $56.25 \%$ were Sprague-Dawley rats. Five studies described the effects of low molecular weight heparin (LMWH, enoxaparin) use. Antiplatelet agents (aspirin and clopidogrel) and NOACs (argatroban and rivaroxaban) were applied in five and three studies, respectively. More than one-half of the studies
(68.75\%) reported joint administration of ACs during animal modeling.

3.2. Study Quality and Risk of Bias Assessments. The risk of bias was evaluated in all 16 studies (Figure 2 and Table S3). Due to poor reporting, most items were viewed as unclear. In assessing selection bias (items 1-3), authors of eight publications merely mentioned randomization, without detailing their procedures; and no authors described allocation concealment. As for performance bias assessment (items 4 and 5), animals were randomly assigned in nine studies, a blinded process cited in one report only. None of the studies mentioned measures for detection bias (item 6 and 7), and most $(81.25 \%)$ were unclear in terms of attrition bias (item 8 ). None of the studies outlined a study protocol, so the risk of reporting bias (item 9) was unclear. As shown in Figure 2, three and eight authors referred to randomization at points of outcome assessment (item 10) and allocation (item 11), respectively. Finally, five of these 16 publications $(31.25 \%)$ did not specify sources of funding (item 12), and conflict of interest declarations (item 13) were largely unclear (43.75\%), as opposed to denials (50\%) or acknowledgments $(6.25 \%)$.

3.3. Animal Survival and Effect Estimates. A total of 16 comparisons involving 390 animals were pooled to investigate animal survival after use of ACs, including low molecular weight heparins (LMWHs) and antiplatelet agent (aspirin) [22, 28-30]. As shown in Figure 3(a), administration of ACs conferred no change in animal survival (risk ratio $[R R]=1.03,95 \%$ confidence interval $(C I): 0.94 \sim 1.13)$, with moderate certainty; and heterogeneity was very low $\left(I^{2}=0 \%\right)$ across all studies. Using subgroup analyses, LMWHs (RR 0.96, 95\% CI 0.84 1.09) and aspirin (RR 1.17, 95\% CI 0.90 1.51) also did not impact animal survival and heterogeneity was very low $\left(I^{2}=0 \%\right)$ across all studies.

3.4. Measures of Hepatic Fibrosis and Effect Estimates. Fibrosis evaluations included semiquantitative METAVIR scores and visual gauging of collagen (type I and III) depositions. To assess hepatic fibrosis, eight comparisons involving 120 animals were pooled. Outcomes suggested amelioration in degrees of fibrosis by AC therapies (enoxaparin and aspirin) (RR 0.66, 95\% CI 0.47 0.94), with very low certainty; and heterogeneity was moderate $\left(I^{2}=71 \%\right)[28,29,31]$ (Figure 3(b) and Table 2). Subgroup analyses were conducted to examine potential factors contributing to the heterogeneity of total events. All subgroups (see Table 3 ), including various animal models, species, and treatment parameters (duration, timing, and types of AC), were sources of heterogeneity, for example, the effect sizes of male species $(\mathrm{RR}=0.65,95 \% \mathrm{CI}$ : $\left.0.4 \sim 1.04 ; I^{2}=79 \%\right)$, and antiplatelet agent subgroup $\left(\mathrm{RR}=0.58\right.$, 95\% CI $\left.0.37 \sim 0.91 ; I^{2}=61 \%\right)$. We further addressed the effect of each anticoagulation separately and found that aspirin has a protective effect on degrees of fibrosis $\left(\mathrm{RR}=0.58,95 \%\right.$ CI $\left.0.37 \sim 0.91 ; I^{2}=61 \%\right)$ that is not 


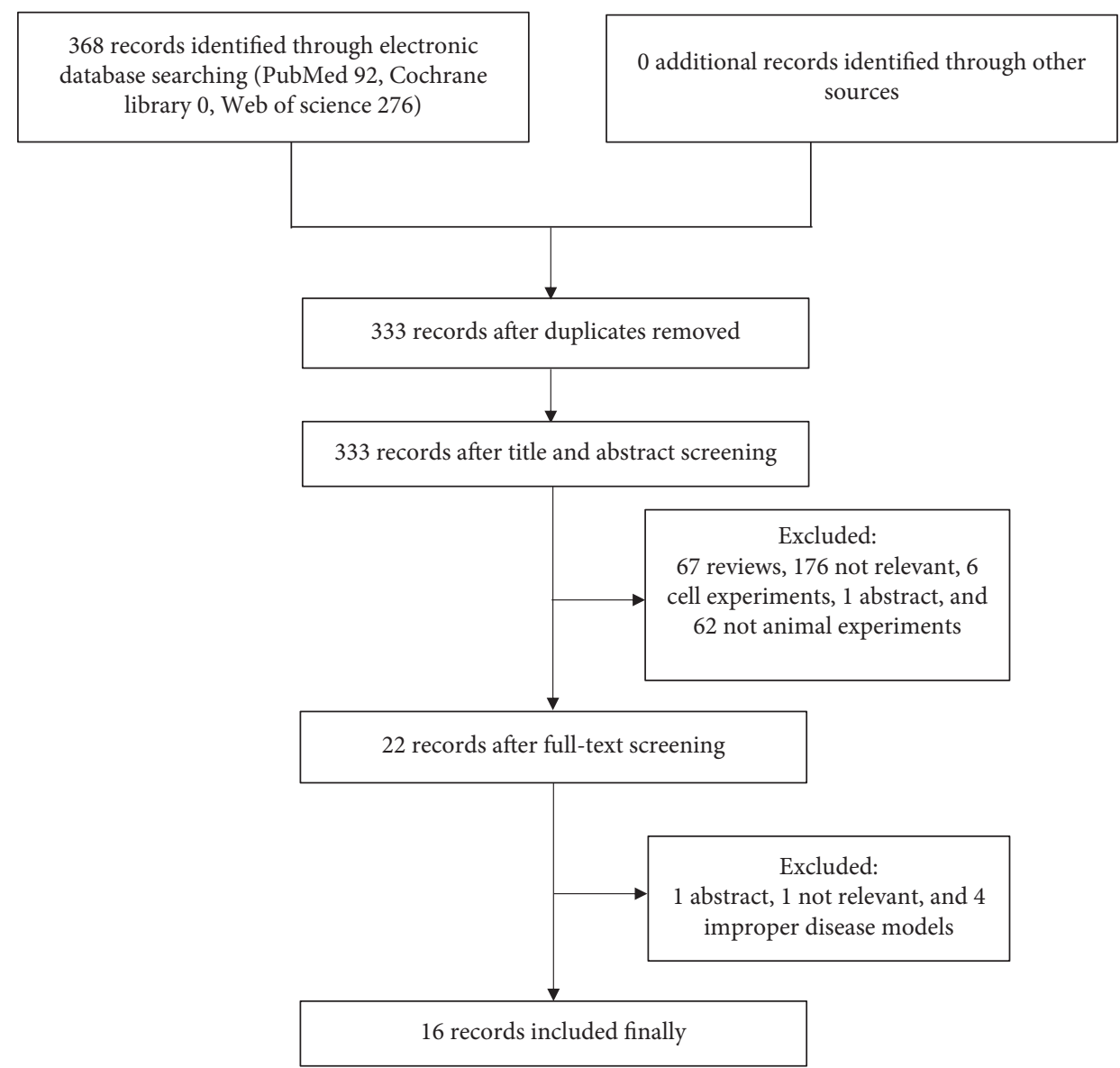

Figure 1: Flow diagram of study selection, searching PubMed, Cochrane Library, and Web of Science (up to November 2020) for studies assessing anticoagulant administration in animal models of chronic liver diseases (total of 16 qualifying publications).

TABlE 1: Characteristics of included studies.

\begin{tabular}{|c|c|c|c|c|c|c|c|}
\hline Study & Study design & Animals & $\begin{array}{l}\text { Animal } \\
\text { number }\end{array}$ & $\begin{array}{c}\text { Modeling } \\
\text { method }\end{array}$ & Treatment groups & Main outcomes & Courses \\
\hline $\begin{array}{l}\text { Kassel et al. } \\
{[12]}\end{array}$ & Unclear & $\mathrm{C} 57 \mathrm{BL} / 6$ & Unclear & $\begin{array}{l}\text { Western diet } \\
\text { (40\% kcal from } \\
\text { milk fat), } \\
\text { NAFLD }\end{array}$ & $\begin{array}{l}\text { Vehicle pumps and } \\
\text { argatroban pumps }\end{array}$ & Antifibrotic effect & $\begin{array}{c}23 \\
\text { weeks }\end{array}$ \\
\hline Li et al. [13] & Unclear & $\begin{array}{c}\text { Sprague- } \\
\text { Dawley rats }\end{array}$ & 52 & $\begin{array}{l}\mathrm{CCl}_{4} / \text { porcine } \\
\text { serum, hepatic } \\
\text { fibrosis }\end{array}$ & $\begin{array}{c}\text { Normal control, } \mathrm{CCl}_{4} \text {, } \\
\text { porcine serum, } \\
\mathrm{CCl} 4+\mathrm{LAAH}, \text { and porcine } \\
\text { serum + LAAH }\end{array}$ & $\begin{array}{l}\text { Liver function tests, and } \\
\text { area of collagens }\end{array}$ & $\begin{array}{c}10 \\
\text { weeks }\end{array}$ \\
\hline $\begin{array}{l}\text { Lee et al. } \\
{[16]}\end{array}$ & Unclear & $\begin{array}{l}\text { Sprague- } \\
\text { Dawley rats }\end{array}$ & 24 & $\begin{array}{c}1 \% \text { DMN, } \\
\text { hepatic fibrosis }\end{array}$ & $\begin{array}{c}\text { Control, DMN, DMN + LH, } \\
\text { and DMN + LHP }\end{array}$ & Antifibrotic effect & 4 weeks \\
\hline $\begin{array}{l}\text { Vilaseca } \\
\text { et al. [17] }\end{array}$ & $\begin{array}{l}\text { Randomized } \\
\text { controlled } \\
\text { study }\end{array}$ & $\begin{array}{l}\text { Wistar rats, } \\
\text { Sprague- } \\
\text { Dawley rats }\end{array}$ & Unclear & $\begin{array}{c}\mathrm{CCl}_{4} / \mathrm{TAA} \\
\text { cirrhosis }\end{array}$ & Rivaroxaban and vehicle & $\begin{array}{l}\text { Antifibrotic effects, HSC } \\
\text { activation, and portal } \\
\text { pressure }\end{array}$ & $\begin{array}{c}18 \\
\text { weeks }\end{array}$ \\
\hline $\begin{array}{l}\text { Cerini et al. } \\
{[21]}\end{array}$ & $\begin{array}{l}\text { Randomized } \\
\text { controlled } \\
\text { study }\end{array}$ & $\begin{array}{l}\text { Wistar rats } \\
\text { and } \\
\text { Sprague- } \\
\text { Dawley rats }\end{array}$ & Unclear & $\begin{array}{l}\mathrm{CCl}_{4} / \mathrm{TAA}, \\
\text { cirrhosis }\end{array}$ & Enoxaparin and vehicle & $\begin{array}{l}\text { Antifibrotic effect and } \\
\text { inflammation response }\end{array}$ & $\begin{array}{c}15 \\
\text { weeks }\end{array}$ \\
\hline $\begin{array}{l}\text { Fortea et al. } \\
{[22]}\end{array}$ & Unclear & $\begin{array}{l}\text { Sprague- } \\
\text { Dawley rats }\end{array}$ & Unclear & $\mathrm{CCl}_{4}$, cirrhosis & $\begin{array}{l}\text { Saline, } \mathrm{CCl}_{4}+\text { Saline, and } \\
\mathrm{CCl}_{4}+\text { enoxaparin }\end{array}$ & $\begin{array}{l}\text { Survival, liver function } \\
\text { tests, antifibrotic effect, } \\
\text { and inflammation } \\
\text { response }\end{array}$ & $\begin{array}{c}12 \\
\text { weeks }\end{array}$ \\
\hline $\begin{array}{l}\text { Assy et al. } \\
{[28]}\end{array}$ & Unclear & $\begin{array}{l}\text { Sprague- } \\
\text { Dawley rats }\end{array}$ & 28 & $\begin{array}{l}\text { TAA, hepatic } \\
\text { cirrhosis }\end{array}$ & $\begin{array}{l}\text { Controls, aspirin, and } \\
\text { enoxaparin }\end{array}$ & $\begin{array}{l}\text { Survival, liver function } \\
\text { tests, and antifibrotic } \\
\text { effect fibrosis }\end{array}$ & 5 weeks \\
\hline
\end{tabular}


TABLE 1: Continued.

\begin{tabular}{|c|c|c|c|c|c|c|c|}
\hline Study & Study design & Animals & $\begin{array}{l}\text { Animal } \\
\text { number }\end{array}$ & $\begin{array}{l}\text { Modeling } \\
\text { method }\end{array}$ & Treatment groups & Main outcomes & Courses \\
\hline Li et al. [29] & $\begin{array}{l}\text { Randomized } \\
\text { controlled } \\
\text { study }\end{array}$ & $\begin{array}{l}\text { Sprague- } \\
\text { Dawley rats }\end{array}$ & 45 & $\begin{array}{l}\text { TAA, hepatic } \\
\text { fibrosis }\end{array}$ & $\begin{array}{c}\text { TAA, TAA + low-dose } \\
\text { aspirin, TAA + high-dose } \\
\text { aspirin, and } \\
\text { TAA + enoxaparin }\end{array}$ & $\begin{array}{l}\text { Liver function tests and } \\
\text { antifibrotic effect }\end{array}$ & 4 weeks \\
\hline $\begin{array}{l}\text { Yan et al. } \\
{[30]}\end{array}$ & $\begin{array}{l}\text { Randomized } \\
\text { controlled } \\
\text { study }\end{array}$ & C57BL/6 & Unclear & $\mathrm{CCl}_{4}$, cirrhosis & $\begin{array}{l}\text { Different groups of the } \\
\text { enzymatically } \\
\text { depolymerized heparins and } \\
\text { saline }\end{array}$ & $\begin{array}{l}\text { Liver function tests, } \\
\text { antifibrotic effect, and } \\
\text { inflammation response }\end{array}$ & 8 weeks \\
\hline $\begin{array}{l}\text { Fujita et al. } \\
{[31]}\end{array}$ & $\begin{array}{l}\text { Randomized } \\
\text { controlled } \\
\text { study }\end{array}$ & Fischer rats & 344 & $\begin{array}{l}\text { A CDAA diet } \\
\text { or an HF/HC } \\
\text { diet, NAFLD }\end{array}$ & $\begin{array}{l}\text { CDAA, CDAA + aspirin, } \\
\text { CDAA + ticlopidine, } \\
\text { CDAA + cilostazol, CSAA }\end{array}$ & $\begin{array}{l}\text { Antifibrotic effect and } \\
\text { inflammation response }\end{array}$ & $\begin{array}{c}16 \\
\text { weeks }\end{array}$ \\
\hline $\begin{array}{l}\text { Abdel- } \\
\text { Salam et al. } \\
{[32]}\end{array}$ & $\begin{array}{l}\text { Randomized } \\
\text { controlled } \\
\text { study }\end{array}$ & $\begin{array}{l}\text { Sprague- } \\
\text { Dawley rats }\end{array}$ & 48 & $\begin{array}{l}\text { Bile duct } \\
\text { ligated (BDL), } \\
\text { cholestatic } \\
\text { liver injury }\end{array}$ & $\begin{array}{c}\text { Sham, BDL, BDL + UFH, } \\
\text { BDL + nadroparin, } \\
\text { BDL + tinzaparin, and } \\
\text { BDL + enoxaparin }\end{array}$ & Liver function tests & 3 weeks \\
\hline $\begin{array}{l}\text { Abe et al. } \\
{[33]}\end{array}$ & Unclear & Wistar rats & Unclear & $\begin{array}{l}\mathrm{CCl}_{4} \text {, hepatic } \\
\text { fibrosis }\end{array}$ & $\mathrm{CCl}_{4}$ and $\mathrm{CCl}_{4}+$ dalteparin & Antifibrotic effect & 7 weeks \\
\hline $\begin{array}{l}\text { Lee et al. } \\
{[34]}\end{array}$ & Unclear & $\begin{array}{c}\text { Sprague- } \\
\text { Dawley rats }\end{array}$ & 24 & $\begin{array}{l}\text { TAA, hepatic } \\
\text { fibrosis }\end{array}$ & $\begin{array}{l}\text { Saline, and dabigatran } \\
\text { etexilate }\end{array}$ & $\begin{array}{c}\text { Liver function tests, } \\
\text { antifibrotic effect, fibrin } \\
\text { deposition, intrahepatic } \\
\text { angiogenesis, and portal } \\
\text { hypertension }\end{array}$ & $\begin{array}{c}12 \\
\text { weeks }\end{array}$ \\
\hline $\begin{array}{l}\text { Mahmoud } \\
\text { et al. [35] }\end{array}$ & $\begin{array}{l}\text { Randomized } \\
\text { controlled } \\
\text { study }\end{array}$ & Albino rats & 24 & $\begin{array}{l}\mathrm{CCl}_{4} \text {, hepatic } \\
\text { fibrosis }\end{array}$ & $\begin{array}{l}\text { Control group, } \mathrm{CCl}_{4} \text {, and } \\
\mathrm{CCl}_{4}+\text { rivaroxaban }\end{array}$ & $\begin{array}{l}\text { Liver function tests, } \\
\text { antifibrotic effect, and } \\
\text { inflammation response }\end{array}$ & 6 weeks \\
\hline $\begin{array}{l}\text { Mahmoud } \\
\text { et al. [36] }\end{array}$ & $\begin{array}{l}\text { Randomized } \\
\text { controlled } \\
\text { study }\end{array}$ & Albino rats & 56 & $\begin{array}{l}\mathrm{CCl}_{4} \text {, hepatic } \\
\text { fibrosis }\end{array}$ & $\begin{array}{l}\text { Normal control, fibrosis } \\
\text { control, dabigatran-treated, } \\
\text { and clopidogrel-treated } \\
\text { group }\end{array}$ & $\begin{array}{l}\text { Liver function tests, } \\
\text { antifibrotic effect, and } \\
\text { inflammation response }\end{array}$ & 6 weeks \\
\hline $\begin{array}{l}\text { Liu et al. } \\
\text { [37] }\end{array}$ & Unclear & $\begin{array}{l}\text { Sprague- } \\
\text { Dawley rats }\end{array}$ & 27 & $\begin{array}{l}\mathrm{CCl}_{4} \text {, hepatic } \\
\text { fibrosis }\end{array}$ & $\begin{array}{l}\text { Control group, } \mathrm{CCl}_{4} \text {, and } \\
\qquad \mathrm{CCl}_{4}+\text { aspirin }\end{array}$ & $\begin{array}{l}\text { Liver function tests, } \\
\text { antifibrotic effect, and } \\
\text { inflammation response }\end{array}$ & 6 weeks \\
\hline
\end{tabular}

ATIII, antithrombin III; BDL, bile duct ligated; CDAA, choline-deficient, L-amino acid-defined; $\mathrm{CCl}_{4}$, carbon tetrachloride; CSAA, choline-sufficient l-amino acid; HF/HC, high-fat high-calorie; L-amino acid-defined; DMN, dimethylnitrosamine; LAAH, low anticoagulant activity heparin; LH, low molecular weight heparin; LHP, low molecular weight heparinepluronic nanogel; NAFLD, nonalcoholic fatty liver disease; TAA, thioacetamide; UFH, unfractionated heparin.

seen with enoxaparin $(\mathrm{RR}=0.83,95 \%$ CI $0.50 \sim 1.40$; $\left.I^{2}=76 \%\right)$.

There were eight comparisons involving 262 animals to assess the effects of ACs on collagen deposition. ACs failed to reduce areas of collagen deposition (mean difference $(\mathrm{MD})=-4.10,95 \% \mathrm{CI}:-12.42 \sim 4.23)$, with very low certainty $[13,22,31,32]$; and heterogeneity was high $\left(I^{2}=98 \%\right)$ (Figure 3(c) and Table 2). Among all types of ACs, standard heparin, LMWHs, and antiplatelet agents were involved in evaluating the effects of ACs on collagen deposition; separate outcome suggested that only standard heparin $\left(\mathrm{MD}=-986.86\right.$, 95\% CI $\left.-1758.75 \sim-214.98, I^{2}=99 \%\right)$ reduced areas of collagen deposition, whereas LMWHs $\left(\mathrm{MD}=1.63,95 \% \mathrm{CI}-6.23 \sim-9.49, I^{2}=96 \%\right)$ and antiplatelet agents $(\mathrm{MD}=-0.07,95 \% \mathrm{CI}-0.41 \sim 0.27)$ did not.

Nine publications $[12,17,21,30,33-37]$ stated that chronicled hepatic fibrosis observed significant attenuation in expression levels of alpha- 1 type 1 collagen (COL1A1), TGF- $\beta 1$, tissue inhibitor of metalloproteinase-1 (TIMP-1), matrix metalloproteinase-2 (MMP-2), and $\alpha$-smooth muscle actin ( $\alpha$-SMA) owing to ACs, such as LMWH (e.g., enoxaparin), NOACs (argatroban and rivaroxaban), thrombin inhibitor (dabigatran), and antiplatelet agent (aspirin). In general, administration of ACs alleviated degrees of hepatic fibrosis in these animal models, proving particularly effective for lowering METAVIR scores and other makers of fibrosis.

3.5. Portal Pressure and Effect Estimates. Eight comparisons involving 180 animals addressed the effects of ACs on PP, showing that $\mathrm{ACs}$ reduced $\mathrm{PP}(\mathrm{MD}=-1.39,95 \% \mathrm{CI}$ $-2.33 \sim-0.44)$, with low certainty; and heterogeneity was low $\left(I^{2}=45 \%\right)$ [17, 21, 22] (Figure 3(d) and Table 2). All subgroups similarly introduced heterogeneity (Table 4), with one example being the effect size of male species $\left(\mathrm{MD}=-1.25,95 \% \mathrm{CI}-2.27 \sim-0.23 ; I^{2}=56 \%\right)$ and LMWH $\left(\mathrm{MD}=-1.25,95 \% \mathrm{CI}-2.27 \sim-0.23 ; I^{2}=56 \%\right)$. In all types of ACs, LMWH (enoxaparin) and NOAC (rivaroxaban) were involved in evaluating the association between ACs and PP, and the separate outcome showed that enoxaparin $\left(\mathrm{MD}=-1.25,95 \%\right.$ CI $\left.-2.27 \sim-0.23, I^{2}=56 \%\right)$ but not rivaroxaban $\left(\mathrm{MD}=-3.28,95 \% \mathrm{CI}-7.11 \sim 0.56, I^{2}=0 \%\right)$ reduced PP. 
(1) Was the allocation sequence adequately generated and applied? (2) Were the groups similar at baseline or were they adjusted for confounders in the analysis?

(3) Was the allocation to the different groups adequately concealed during?

(4) Were the animals randomly housed during the experiment?

(5) Were the caregivers and/or investigators blinded from knowledge which intervention each animal received during the experiment?

(6) Were animals selected at random for outcome assessment?

(7) Was the outcome assessor blinded?

(8) Were incomplete outcome data adequately addressed?

(9) Are reports of the study free of selective outcome reporting?

(10) Was the study stated randomization at outcome assessment?

(11) Was the study stated randomization at allocation level?

(12) Was the study state the source of funding?

(13) Was there conflict of interest?

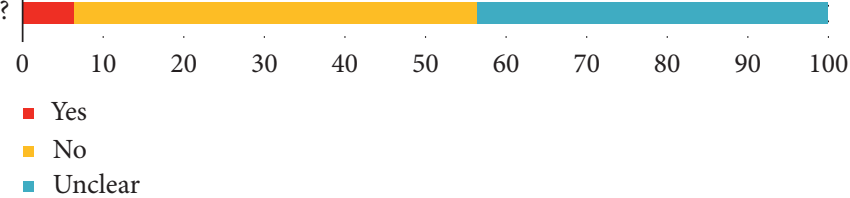

Figure 2: Risk of bias assessments for individual animal studies depicted in a bar chart showing the percentage of all studies that met each quality item, scored as "Yes," "No," or "Unclear."

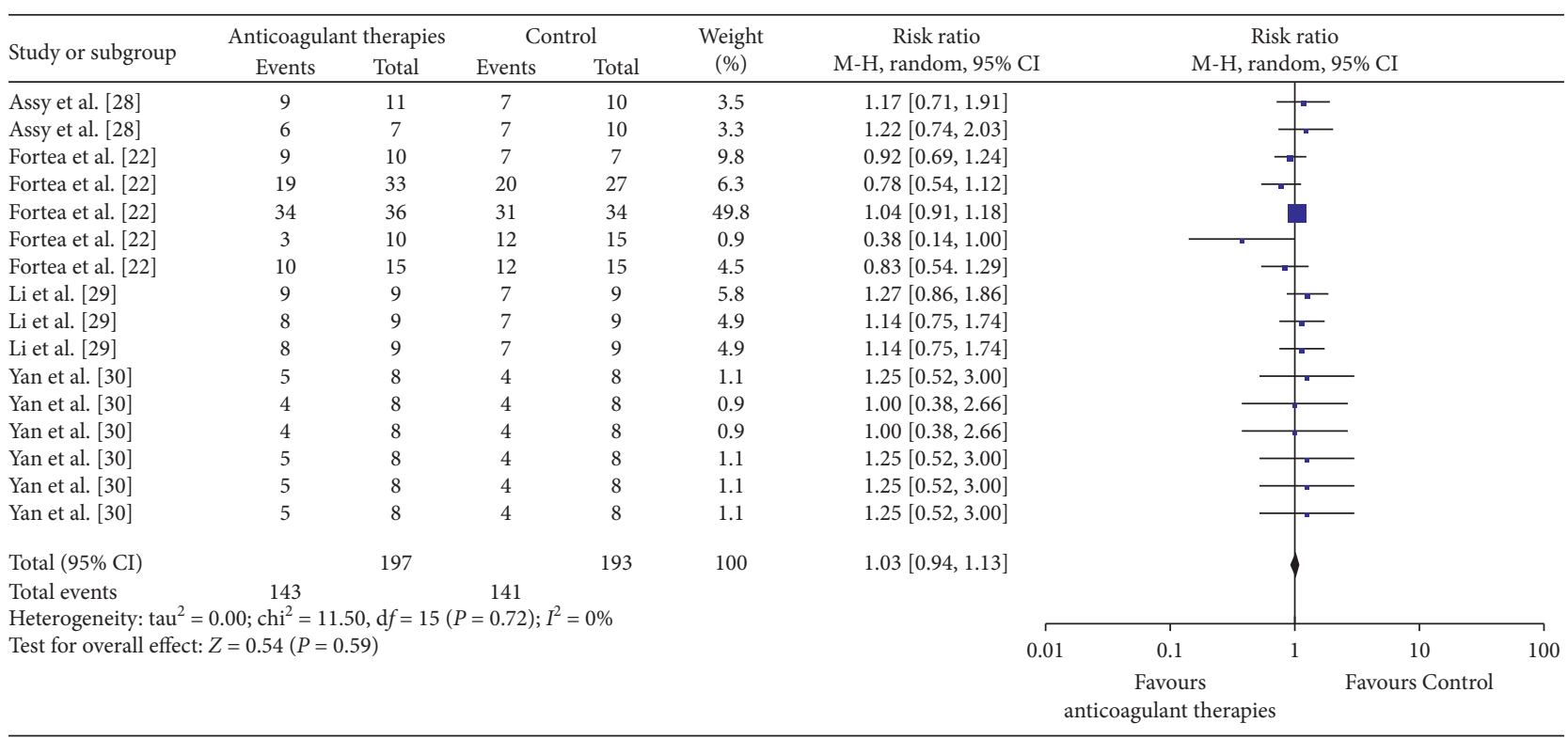

(a)

FIgURE 3: Continued. 


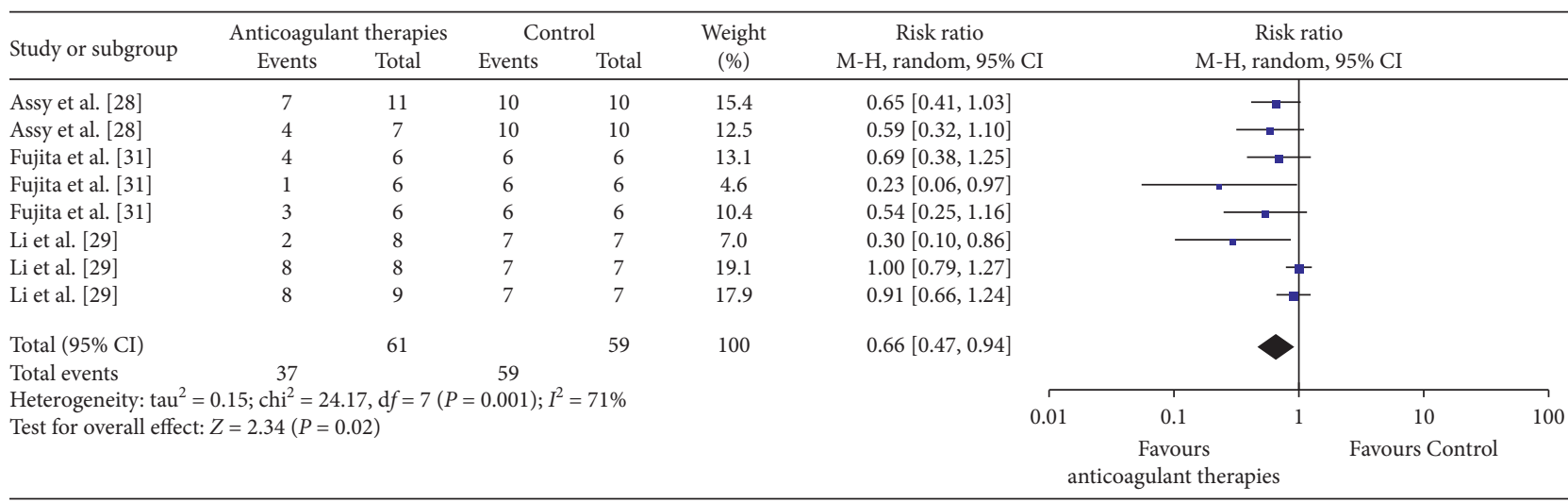

(b)

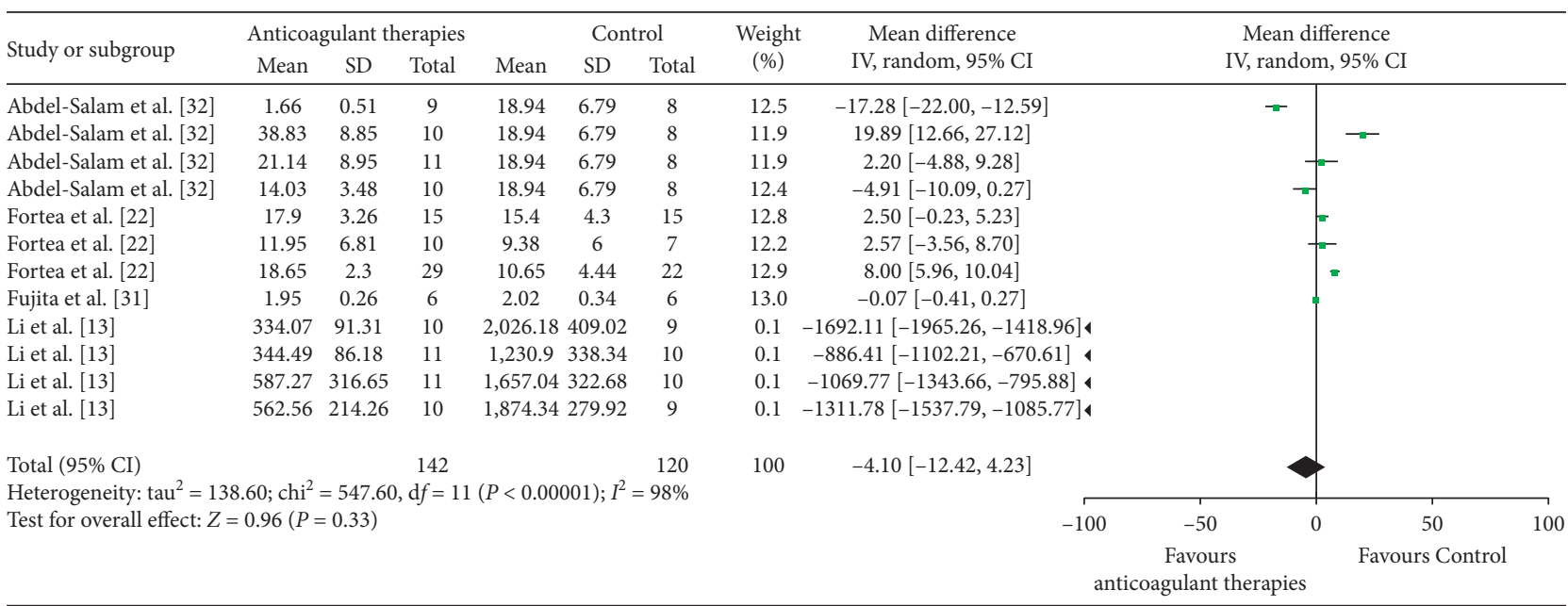

(c)

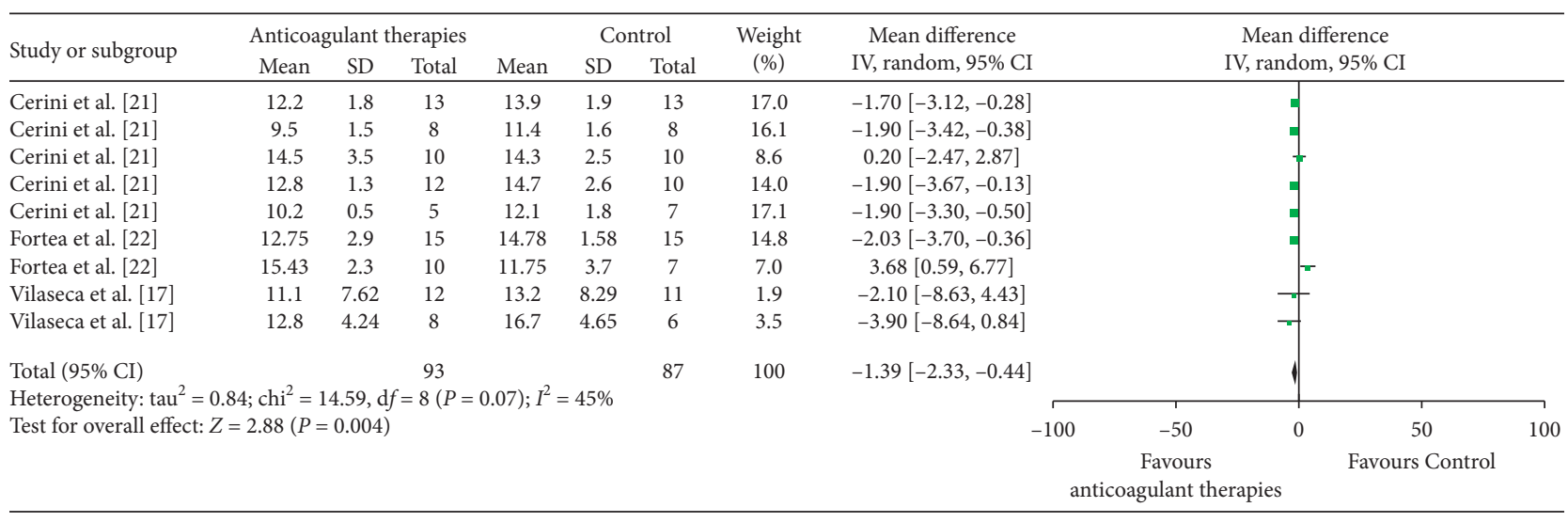

(d)

FIGURE 3: Forest plots in animal models of chronic liver diseases, comparing (a) animal survival, (b) METAVIR fibrosis scores, (c) collagen deposition, and (d) portal pressures after anticoagulant administration.

3.6. Inflammation (Serum TNF- $\alpha$ ) and Effect Estimates. Four comparisons involving 48 animals were analyzed to assess the effects of ACs (antiplatelet agents) on serum levels of tumor necrosis factor-alpha (TNF- $\alpha$ ), which was lower in antiplatelet agents-treated (versus untreated) animals
$(\mathrm{MD}=-169.69,95 \% \mathrm{CI}:-257.64 \sim-81.74)$, with very low certainty [31]; and heterogeneity was high $\left(I^{2}=80 \%\right)$ (Figure 4(a) and Table 2).

Nine studies reported on hepatic inflammatory responses to AC treatment, five of them showing that ACs 


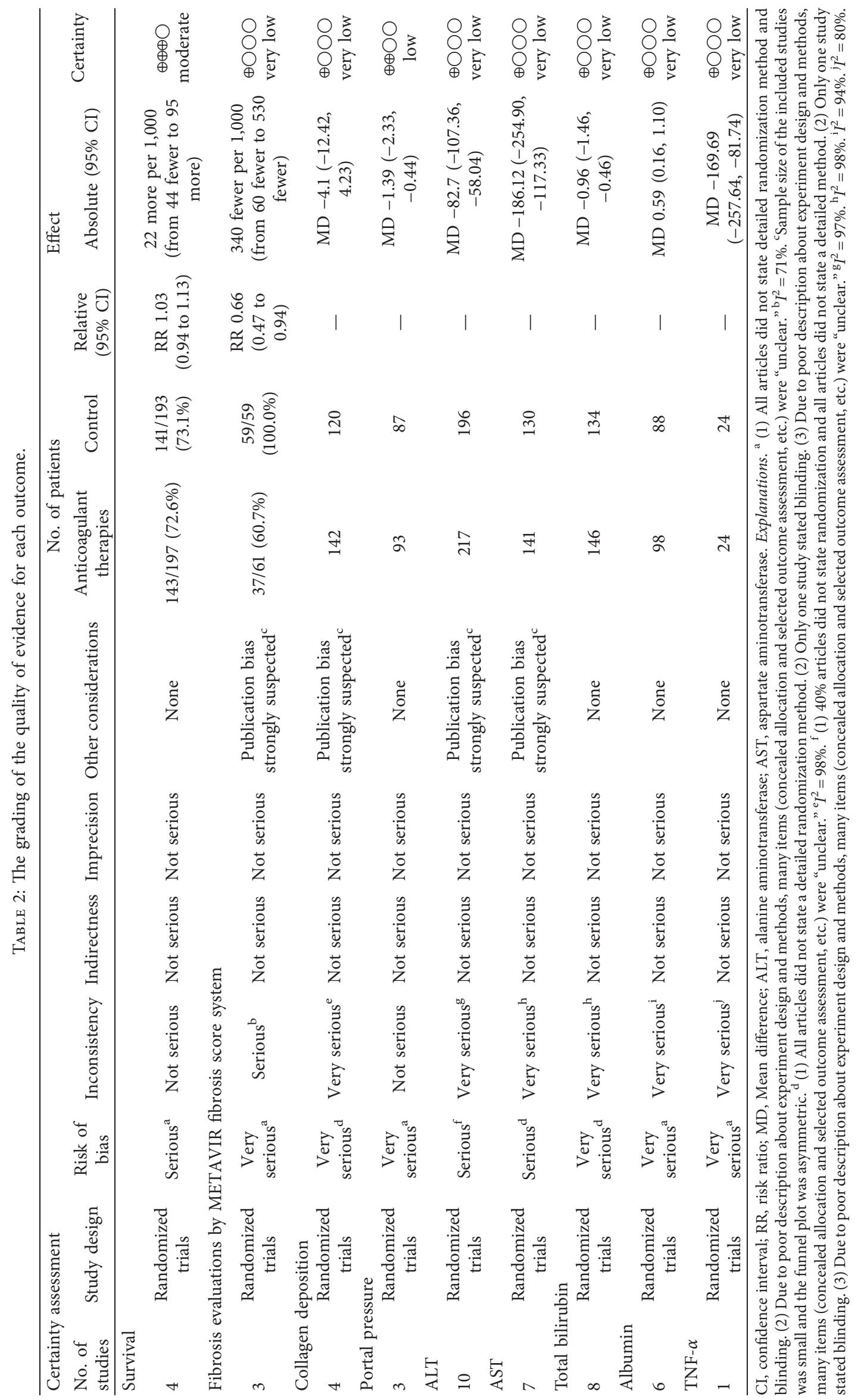


TABLE 3: Subgroup analyses for fibrosis evaluation indicated by the METAVIR fibrosis score system.

\begin{tabular}{|c|c|c|c|c|c|}
\hline \multirow[t]{2}{*}{ Group/subgroup } & \multirow[t]{2}{*}{ Weight (\%) } & \multicolumn{2}{|c|}{ Effect size } & \multicolumn{2}{|c|}{$\begin{array}{l}\text { Heterogeneity for } \\
\text { each subgroup }\end{array}$} \\
\hline & & RR & $95 \% \mathrm{CI}$ & $I^{2}(\%)$ & $p$ \\
\hline All experiments & 100 & 0.66 & $(0.47,0.94)$ & 71 & 0.001 \\
\hline \multicolumn{6}{|l|}{ Animal model } \\
\hline Liver fibrosis/cirrhosis & 71.9 & 0.73 & $(0.5,1.07)$ & 74 & 0.004 \\
\hline Nonalcoholic fatty liver disease & 28.1 & 0.55 & $(0.33,0.93)$ & 19 & 0.29 \\
\hline \multicolumn{6}{|l|}{ Animal species } \\
\hline Male & 72.1 & 0.65 & $(0.4,1.04)$ & 79 & 0.0002 \\
\hline Unclear & 27.9 & 0.63 & $(0.44,0.91)$ & 0 & 0.79 \\
\hline \multicolumn{6}{|l|}{ Treatment duration (weeks) } \\
\hline$\leq 8$ & 71.9 & 0.73 & $(0.5,1.07)$ & 74 & 0.004 \\
\hline$>8$ & 28.1 & 0.55 & $(0.33,0.93)$ & 19 & 0.29 \\
\hline \multicolumn{6}{|l|}{ Treatment timing } \\
\hline Simultaneous injection at model induction & 56.0 & 0.61 & $(0.46,0.81)$ & 0 & 0.65 \\
\hline Injection after model induction & 44.0 & 0.79 & $(0.46,1.35)$ & 81 & 0.005 \\
\hline \multicolumn{6}{|l|}{ Anticoagulation type } \\
\hline Low molecular weight heparin (enoxaparin) & 34.5 & 0.83 & $(0.5,1.4)$ & 76 & 0.04 \\
\hline Antiplatelet agents (aspirin, ticlopidine and cilostazol) & 65.5 & 0.58 & $(0.37,0.91)$ & 61 & 0.03 \\
\hline
\end{tabular}

CI, confidence interval; RR, risk ratio.

TABLE 4: Subgroup analyses for portal pressure.

\begin{tabular}{|c|c|c|c|c|c|}
\hline \multirow[t]{2}{*}{ Group/subgroup } & \multirow[t]{2}{*}{ Weight (\%) } & \multicolumn{2}{|c|}{ Effect size } & \multicolumn{2}{|c|}{$\begin{array}{l}\text { Heterogeneity for } \\
\text { each subgroup }\end{array}$} \\
\hline & & $\mathrm{MD}$ & $95 \% \mathrm{CI}$ & $I^{2}(\%)$ & $p$ \\
\hline All experiments & 100 & -1.39 & $(-2.33,-0.44)$ & 45 & 0.07 \\
\hline \multicolumn{6}{|l|}{ Animal model } \\
\hline Liver cirrhosis & 100 & -1.39 & $(-2.33,-0.44)$ & 45 & 一 \\
\hline Other & 0 & - & - & - & - \\
\hline \multicolumn{6}{|l|}{ Animal species } \\
\hline Male & 94.6 & -1.25 & $(-2.27,-0.23)$ & 56 & 0.03 \\
\hline Unclear & 5.4 & -3.28 & $(-7.11,0.56)$ & 0 & 0.66 \\
\hline \multicolumn{6}{|l|}{ Treatment duration (weeks) } \\
\hline$\leq 8$ & 100 & -1.39 & $(-2.33,-0.44)$ & 45 & - \\
\hline$>8$ & 0 & - & - & - & - \\
\hline \multicolumn{6}{|l|}{ Treatment timing } \\
\hline Simultaneous injection at model induction & 21.8 & 0.67 & $(-4.92,6.26)$ & 90 & 0.001 \\
\hline Injection after model induction & 78.2 & -1.75 & $(-2.46,-1.03)$ & 0 & 0.81 \\
\hline \multicolumn{6}{|l|}{ Anticoagulation type } \\
\hline Low molecular weight heparin (enoxaparin) & 94.6 & -1.25 & $(-2.27,-0.23)$ & 56 & 0.03 \\
\hline Novel oral anticoagulants (rivaroxaban) & 5.4 & -3.28 & $(-7.11,0.56)$ & 0 & 0.66 \\
\hline
\end{tabular}

$\mathrm{CI}$, confidence interval; $\mathrm{MD}$, mean difference.

(argatroban and LMWH) significantly dampened inflammation in the liver $[12,30,35-37]$. Hepatic macrophage and neutrophil accumulation/clustering were diminished as a result of reduced protein expression (CD68, MCP-1, F4/80, ICAM-1, and MIP-2) and cytokine secretion (TNF- $\alpha$, interleukin- (IL-) 6, and IL-1 $\beta$ ). However, the other four studies failed to credit LMWH (dalteparin and enoxaparin) and thrombin inhibitor (dabigatran) with anti-inflammatory effects [21, 22, 33, 34].

3.7. Indices of Hepatic Damage (ALT, AST, Albumin, and Total Bilirubin) and Effect Estimates. Overall, 24 comparisons (in 413 animals) $[13,16,17,21,22,31,32,34-36], 16$ comparisons (in 271 animals) [16, 17, 21, 32, 34-36], 10 comparisons (in 186 animals) [13, 16, 17, 22, 35, 36], and 16 comparisons (in 280 animals) [16, 17, 22, 28, 32, 34-36] were undertaken to investigate the effects of AC fluctuations in alanine aminotransferase (ALT), aspartate aminotransferase (AST), albumin, and total bilirubin, respectively, as markers of hepatic injury. Pooled analyses indicated that in ACtreated (versus untreated) animals, liver damage was reduced (ALT: $M D=-82.70,95 \% \mathrm{CI}$ : -107.36 -58.04; AST: $\mathrm{MD}=-186.12, \quad 95 \% \quad \mathrm{CI}: \quad-254.90 \sim-117.33$; albumin: $\mathrm{MD}=0.59,95 \% \mathrm{CI}: 0.16-1.01$; total bilirubin: $\mathrm{MD}=-0.96$, 95\% CI: -1.46 -0.46), with very low certainty. However, heterogeneity was high ( $I^{2}$ : ALT, 97\%; AST, 98\%; albumin 94\%; total bilirubin, 98\%) (Figures 4(b)-4(e) and Table 2). As 


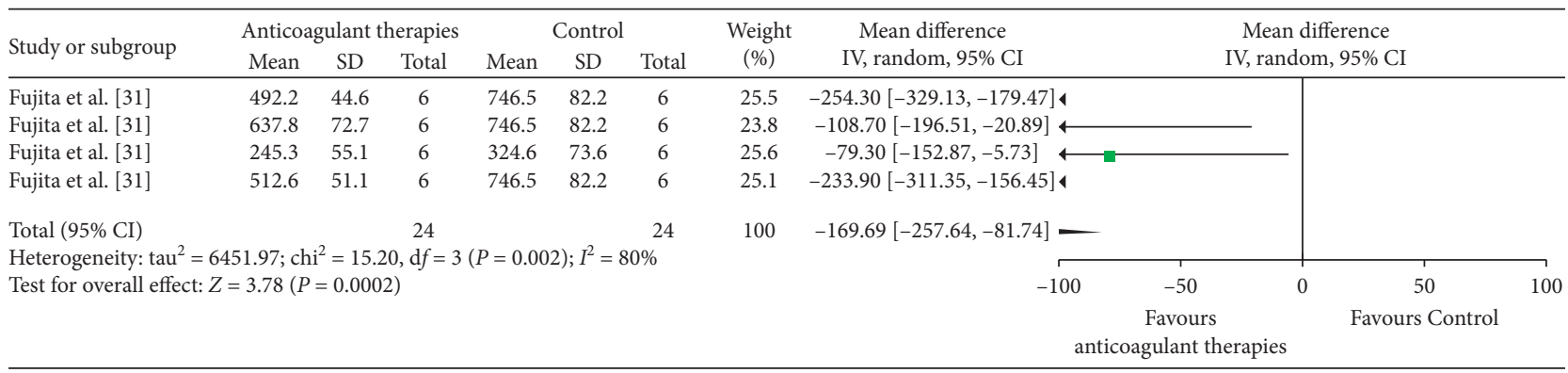

(a)

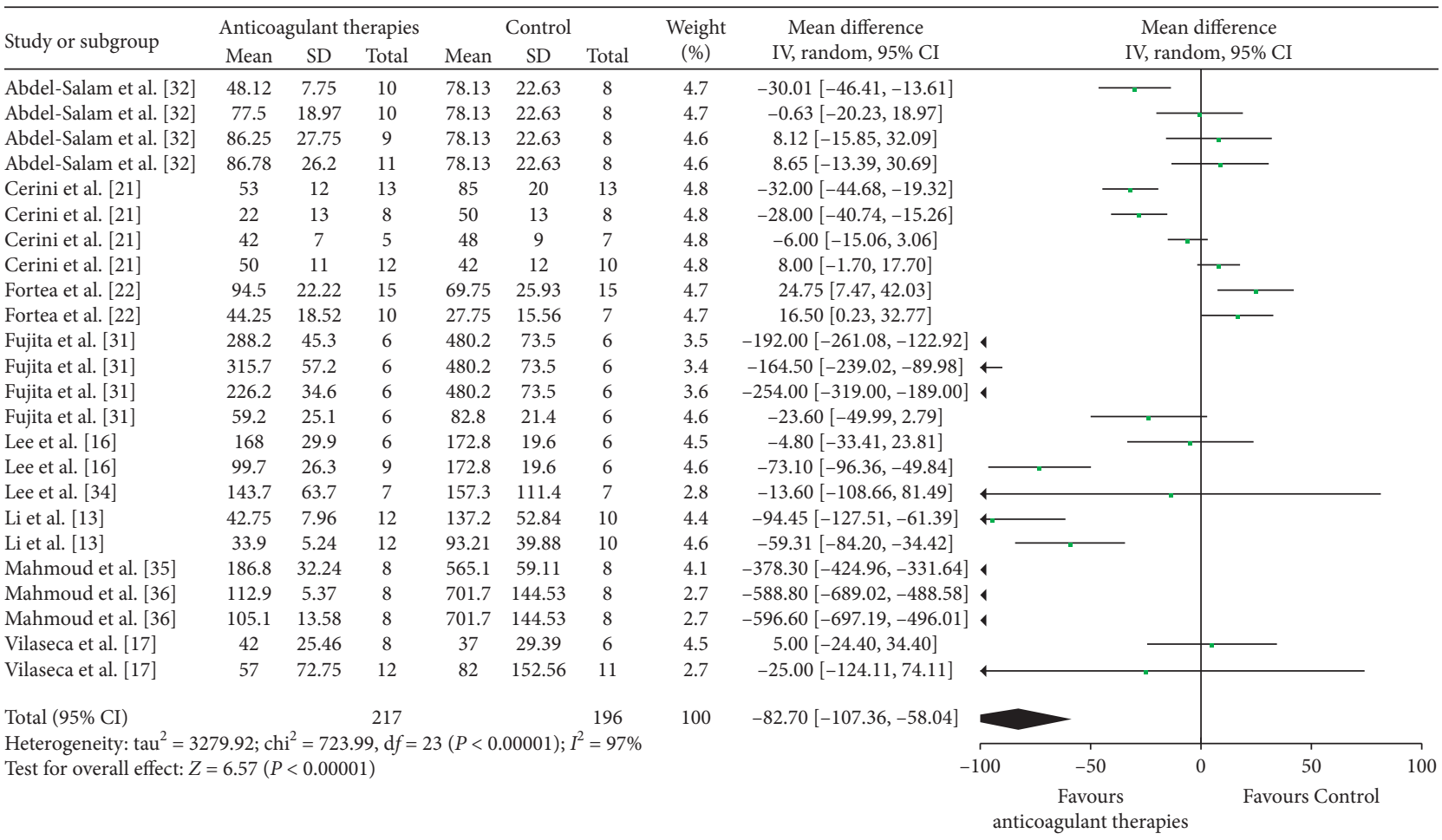

(b)

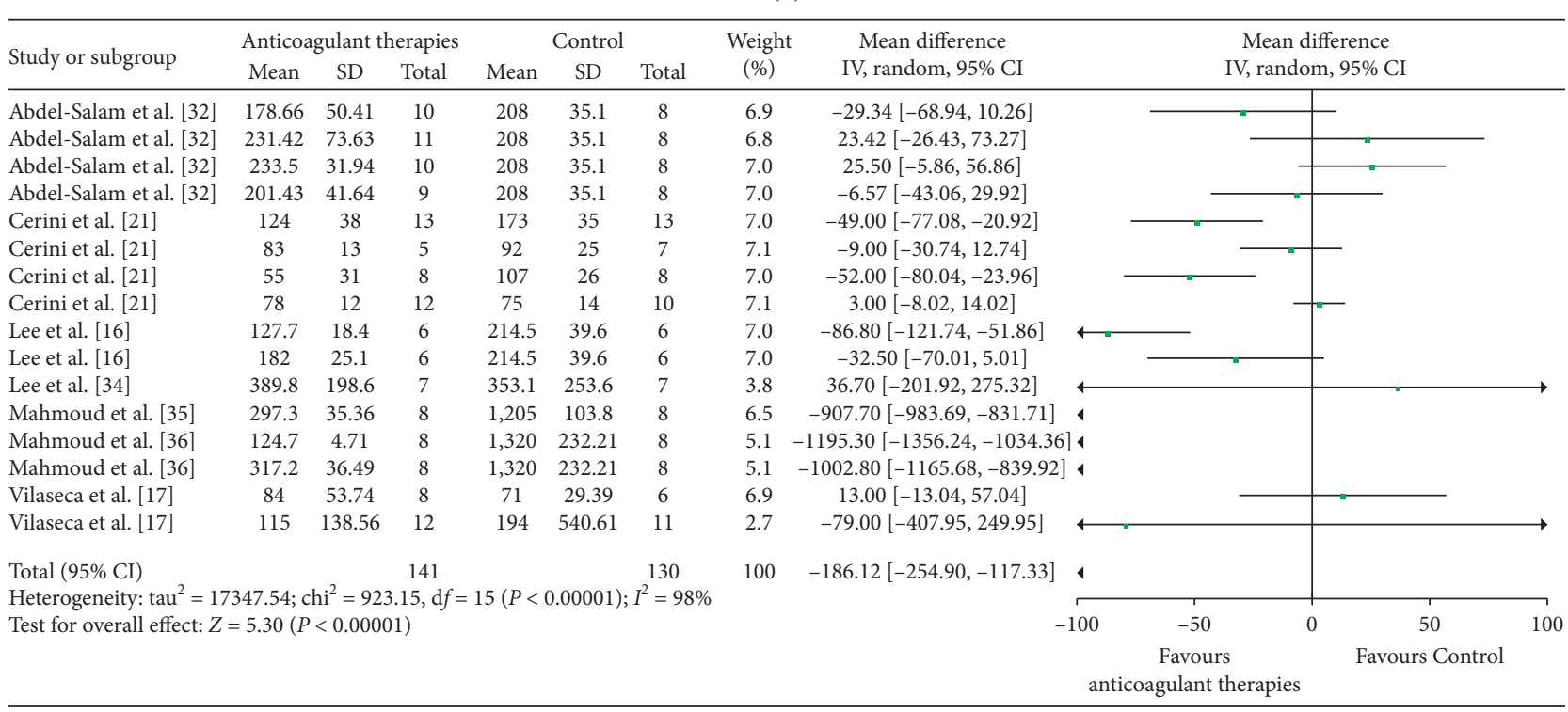

(c)

Figure 4: Continued. 


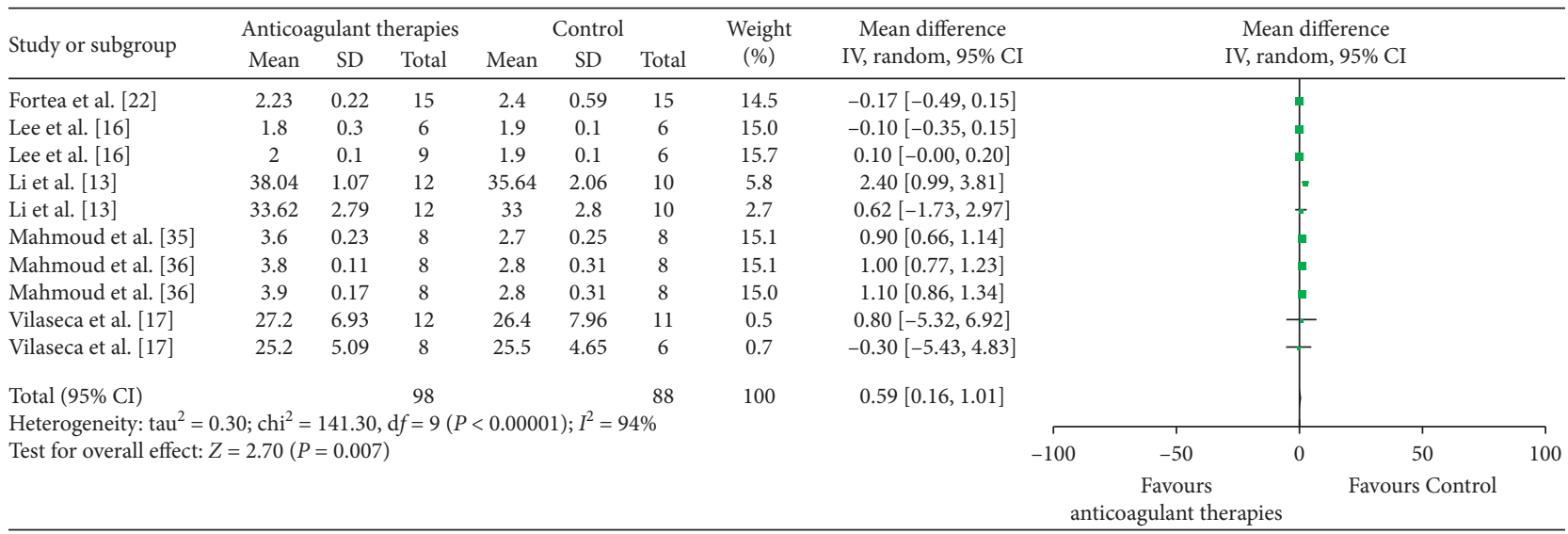

(d)

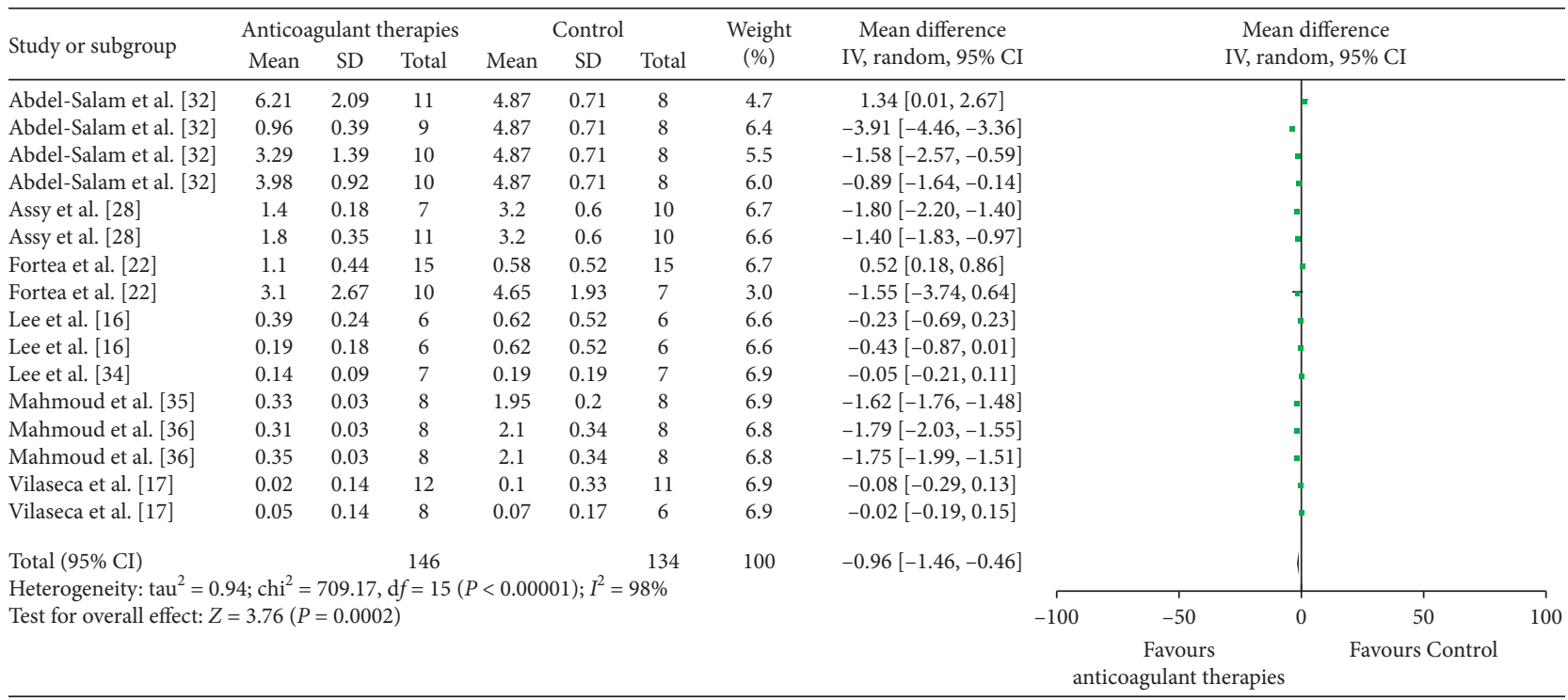

(e)

FIGURE 4: Forest plots in animal models of chronic liver diseases comparing effects of anticoagulant use on serum levels of (a) TNF- $\alpha$, reflecting inflammation, and (b-e) indices of functional hepatic damage (ALT, AST, albumin, and total bilirubin).

TABLE 5: Analyses for the effect of different anticoagulant therapies on the indices of hepatic damage.

\begin{tabular}{|c|c|c|c|c|c|c|}
\hline \multirow{2}{*}{$\begin{array}{l}\text { Anticoagulant } \\
\text { therapies }\end{array}$} & \multicolumn{6}{|c|}{ Effect estimates of indices of hepatic damage } \\
\hline & ALT & & AST & & Albumin & Total bilirubin \\
\hline Antiplatelet agents & $\begin{array}{c}\mathrm{MD}-242.29,95 \% \\
\mathrm{CI}(-409.06 \\
-75.51) \\
\end{array}$ & $p=0.004$ & $\begin{array}{c}\mathrm{MD}-1195.30,95 \% \\
\mathrm{CI}(-1356.24 \\
-1034.36) \\
\end{array}$ & $p<0.001$ & $\begin{array}{l}\text { MD } 1.10,95 \% \\
\text { CI }(0.86,1.34)\end{array} \quad p<0.001$ & $\begin{array}{l}\text { MD }-1.79, \\
\text { 95\% CI } \quad p<0.001 \\
(-2.00,-1.59)\end{array}$ \\
\hline LMWHs & $\begin{array}{l}\mathrm{MD}-10.41,95 \% \\
\mathrm{CI}(-24.45,3.63)\end{array}$ & $p=0.15$ & $\begin{array}{c}\text { MD }-25.17,95 \% \text { CI } \\
(-46.52,-3.83)\end{array}$ & $p=0.02$ & $\begin{array}{c}\mathrm{MD}-0.01 \\
95 \% \mathrm{CI} \\
(-0.19,0.17) \\
\end{array}$ & $\begin{array}{c}\mathrm{MD}-1.16, \\
95 \% \mathrm{CI}(-2.18, \quad p=0.03 \\
-0.13)\end{array}$ \\
\hline $\begin{array}{l}\text { Factor Xa inhibitor } \\
\text { (rivaroxaban) }\end{array}$ & $\begin{array}{c}\mathrm{MD}-133.66,95 \% \\
\mathrm{CI}(-412.73 \\
145.40) \\
\end{array}$ & $p=0.35$ & $\begin{array}{c}\text { MD }-329.49,95 \% \\
\text { CI }(-1053.49 \\
394.52) \\
\end{array}$ & $p=0.37$ & $\begin{array}{l}\operatorname{MD} 0.90,95 \% \\
\text { CI }(0.66,1.13)\end{array} \quad p<0.001$ & $\begin{array}{l}\text { MD }-0.57 \\
95 \% \text { CI }(-1.69, \quad p=0.31 \\
0.54)\end{array}$ \\
\hline Standard heparin & $\begin{array}{c}\mathrm{MD}-47.46,95 \% \\
\mathrm{CI}(-107.27 \\
12.34)\end{array}$ & $p=0.12$ & $\begin{array}{l}\text { MD } 23.42,95 \% \text { CI } \\
\quad(-26.43,73.27)\end{array}$ & $p=0.36$ & $\begin{array}{l}\text { MD } 1.77,95 \% \\
\text { CI }(0.10,3.44)\end{array} \quad p=0.04$ & $\begin{array}{l}\text { MD } 1.34,95 \% \\
\text { CI }(0.01,2.67)\end{array} \quad p=0.05$ \\
\hline Thrombin inhibitor & $\begin{array}{c}\mathrm{MD}-300.97,95 \% \\
\mathrm{CI}(-864.44 \\
262.71) \\
\end{array}$ & $p=0.30$ & $\begin{array}{c}\text { MD }-486.86,95 \% \\
\text { CI }(-1505.52 \\
531.81)\end{array}$ & $p=0.35$ & $\begin{array}{l}\text { MD } 1.00,95 \% \\
\text { CI }(0.77,1.23)\end{array} \quad p<0.001$ & $\begin{array}{c}\mathrm{MD}-0.90 \\
95 \% \mathrm{CI} \\
(-2.56,0.77)\end{array}$ \\
\hline
\end{tabular}

CI, confidence interval; MD, mean difference; LMWHs, low molecular weight heparin; ALT, alanine aminotransferase; AST, aspartate aminotransferase. 
shown in Table 5, we further assessed the effect on indices of hepatic damage of each anticoagulation separately. The data supported that only antiplatelet agents improved all serum indices of hepatic function, including ALT, AST, albumin, and total bilirubin.

3.8. Sensitivity Analysis. Sensitivity analysis was performed to evaluate the robustness of effect sizes for our pooled outcome estimates by excluding single studies sequentially. For example, the findings of Fortea et al. [22] implied that enoxaparin had no impact on collagen-stained areas. By removing this publication, the pooled effect was altered, showing significantly less collagen deposition due to AC therapy $(\mathrm{MD}=-31.01,95 \% \mathrm{CI}:-50.79$ to -11.22$)$, so we must be cautious when interpreting this outcome.

\section{Discussion}

In patients with various chronic liver diseases, coagulopathy-related complications often arise in conjunction with hepatic fibrosis and cirrhosis. Intrahepatic thrombosis and hepatic fibrosis are closely associated conditions [38, 39]. Although ACs are widely used to treat or reduce PVT in patients with cirrhosis [40], the effects of antithrombotic therapies on hepatic fibrosis have yet to be fully examined. Current treatments aimed at fibrotic/cirrhotic states and the concomitant complications remain unsatisfactory. Our meta-analysis of preclinical data provides evidence that AC treatment of animals with chronic liver diseases lowers PP, lessens the progression of fibrosis, reduces hepatic inflammation, and improves hepatic function, compared with vehicle-treated controls, which may thus represent a promising tool in chronic liver diseases. To our knowledge, the present meta-analysis is the first to address AC safety and efficacy in animal models simulating the fibrosis/cirrhosis imposed by various chronic liver diseases.

Hepatitis $\mathrm{C}$ virus (HCV) and hepatitis B virus (HBV), alcohol abuse, and NAFLD were the leading causes of chronic liver diseases worldwide in clinical cases. Ethanol intake appears as an independent predictor of death in patients with chronic viral liver diseases [41]. In contrast, in our experimental analysis, most (81.25\%) models of chronic liver disease were hepatic fibrosis/cirrhosis and were largely induced by $\mathrm{CCl}_{4}(75 \%)$ and TAA $(31.25 \%)$. There was only one model of cholestatic liver injury as well as two models of NAFLD. In cirrhosis, PVT is a frequent complication, particularly in subjects with moderate-severe liver failure, with a prevalence of $17 \%$ [42]. And similar to cirrhosis, NAFLD was also described as the prototypic prothrombotic liver disease due to derangements across all stages of hemostasis, leading to an increased risk of thrombotic events including portal venous and the systemic circulation [4]. In addition, NAFLD may progress to nonalcoholic steatohepatitis- (NASH-) related cirrhosis and appears at higher mortality. Currently, anticoagulant therapy of cirrhosis across the NAFLD spectrum is as yet incompletely defined [5]. Therefore, the experimental model of NAFLD should also ideally be more used in the future to evaluate the impact and safety of anticoagulants.

Either standard or low molecular weight heparin, vitamin $\mathrm{K}$ antagonists, antiplatelet agents, and NOACs are a heterogeneous class of drugs. Here, we addressed the effect on all outcomes of each anticoagulant drug separately. Based upon qualifying preclinical trials, we have determined that all types of AC treatments do not impact animal survival, although antiplatelet agents do lower METAVIR fibrosis scores and standard heparin does reduce areas of collagen deposition. HSCs are one kind of liver-resident populations and generally activated in the context of cirrhosis to secrete an excess of extracellular matrix and intensify hepatic vascular tone, contributing to fibrosis progression and portal hypertension [43]. We have observed significant attenuation in expression levels of activated HSCs markers (e.g., COL1A1, TGF- $\beta 1$, TIMP-1, MMP-2, and $\alpha$-SMA) owing to LMWH (e.g., enoxaparin), NOACs (argatroban and rivaroxaban), thrombin inhibitor (dabigatran), and antiplatelet agent (aspirin). In addition, we found lower PP values in enoxaparin-treated animals than in vehicle-treated controls. It is well known that the fibrotic liver is an inflammatory microenvironment which is characterized by infiltrating various leukocytes (e.g., macrophages and neutrophils). In turn, inflammatory injury drives fibrogenesis [43]. According to our results, we found that hepatic macrophage and neutrophil accumulation/clustering were diminished by argatroban and $\mathrm{LMWH}$ as a result of reduced protein expression (CD68, MCP-1, F4/80, ICAM-1, and MIP-2) and cytokine secretion (TNF- $\alpha$, IL-6, and IL-1 $\beta$ ). Likewise, we noted a dampening of inflammation (a regular accompaniment of hepatic fibrosis) [44] by antiplatelet drugs, reflected in diminished expression of TNF- $\alpha$, and normalized levels of various markers (ALT, AST, albumin, and total bilirubin) signaling preservation of hepatic function. Our outcomes were slightly different from clinical studies in which anticoagulants were mainly applied to evaluate survival, the incidence of bleeding events reporting, partial or complete portal venous system recanalization, and the rate of PVT recurrence in patients with cirrhosis $[9,45]$.

Methodological weaknesses of preclinical studies often result in exaggerated effect sizes [46-48], so we invoked the SYRCLE's risk of bias tool to gauge the quality of these particular efforts. Unfortunately, most checklist items were deemed "unclear" due to inadequate reporting, indicating the poor reporting quality of studies and overestimation of effect sizes. This exercise underscored the need to render adequate quality reporting when conveying preclinical scientific accounts. Moreover, our estimates of effects were tenuous and were rated as very low, low, or moderate certainty in most instances. It is therefore quite likely that subsequent research of this nature will deviate. Furthermore, the heterogeneity of factors in animal models, species, and $\mathrm{AC}$ administration (i.e., duration, timing, and type of AC) limited the generalizability of our evidence. For example, the findings of Fortea et al. [22] indicated that enoxaparin does not ameliorate liver fibrosis in rats, and three separate sources [21, 22, 33] reported similar outcomes for systemic inflammation in both LMWH-treated (enoxaparin and 
dalteparin) and control groups. The results of the three trials differed from those of other studies selected.

Our review has several acknowledged limitations, one being the range of experimental models and different types of injuries represented. Model-dependent mechanisms may certainly impact the conclusions reached. The varied activities and safety profiles of assorted ACs administered were also problematic. A more in-depth investigation into the duration and timing of $\mathrm{AC}$ therapeutics is warranted as a consequence. Likewise, the heterogeneity, uncertainty, and small sample sizes confronted in this meta-analysis undermined the validity of the conclusions gathered. Despite our assertion of safety in dispensing ACs for cirrhosis, its conveyance into clinical practice would constitute a sizeable leap. Animals do not share the compensated/ decompensated phenotypes of humans.

Based on available animal studies, our findings illustrate the potential benefits of anticoagulant therapy in preventing hepatic fibrosis, portal pressure, inflammatory activity, and serum indices of hepatocellular injury, without impacting survival. More high-quality preclinical studies are still needed in this field.

\section{Abbreviations}

AC: Anticoagulant

ALT: $\quad$ Alanine aminotransferase

AST: Aspartate aminotransferase

BDL: Bile duct ligation

$\mathrm{CCl}_{4}$ : Carbon tetrachloride

CDAA: Choline-deficient L-amino acid-defined

DMN: Dimethylnitrosamine

ERK: Extracellular signal-regulated kinase

HSC: Hepatic stellate cell

$\mathrm{HF} / \mathrm{HC}$ : High-fat high-calorie

MMP-2: Matrix metalloproteinase-2

MD: $\quad$ Mean difference

NAFLD: Nonalcoholic fatty liver disease

NO: $\quad$ Nitric oxide

NOAC: Novel direct oral anticoagulant

PVT: $\quad$ Portal vein thrombosis

PP: $\quad$ Portal pressure

RR: $\quad$ Risk ratio

SD: $\quad$ Standard deviation

SEM: $\quad$ Standard error of the mean

TAA: Thioacetamide

TGF- $\beta$ : Transforming growth factor-beta

TIMP-1: Tissue inhibitor of metalloproteinase-1

TNF- $\alpha$ : Tumor necrosis factor-alpha.

\section{Data Availability}

The datasets used in the study are available from the corresponding author upon reasonable request.

\section{Disclosure}

The funders had no role in the design of the study, data collection, and analysis or manuscript writing.

\section{Conflicts of Interest}

The authors declare no conflicts of interest.

\section{Authors' Contributions}

SYC conceived and designed the study. RZ, XQH, YYJ, and JW extracted data and performed the statistical analysis. RZ, $\mathrm{XQH}, \mathrm{YYJ}$, and SYC analyzed the data and wrote the manuscript. All authors read and approved the final manuscript. Rui Zhang, Xiaoquan Huang, and Yingyi Jiang contributed equally to this work.

\section{Acknowledgments}

This work was supported by the Innovation Fund of Science and Technology Commission of Shanghai Municipality (Nos. 15411950501, 15411950507, and 17140902700).

\section{Supplementary Materials}

Table S1: completed PRISMA checklist reporting. Table S2: detailed characteristics of selected publications. Table S3: risk of bias assessment for all studies. (Supplementary Materials)

\section{References}

[1] T. Lisman and R. J. Porte, "Rebalanced hemostasis in patients with liver disease: evidence and clinical consequences," Blood, vol. 116, no. 6, pp. 878-885, 2010.

[2] G. Targher, G. Zoppini, P. Moghetti, and C. Day, "Disorders of coagulation and hemostasis in abdominal obesity: emerging role of fatty liver," Seminars in Thrombosis and Hemostasis, vol. 36, no. 1, pp. 041-048, 2010.

[3] F. Leonardi, N. D. Maria, and E. Villa, "Anticoagulation in cirrhosis: a new paradigm?" Clinical and Molecular Hepatology, vol. 23, no. 1, pp. 13-21, 2017.

[4] M. Spinosa and J. G. Stine, "Nonalcoholic fatty liver diseaseevidence for a thrombophilic state?" Current Pharmaceutical Design, vol. 26, no. 10, pp. 1036-1044, 2020.

[5] S. Ballestri, M. Capitelli, M. C. Fontana et al., "Direct oral anticoagulants in patients with liver disease in the era of nonalcoholic fatty liver disease global epidemic: a narrative review," Advances in Therapy, vol. 37, no. 5, pp. 1910-1932, 2020.

[6] I. R. Wanless, J. J. Liu, and J. Butany, "Role of thrombosis in the pathogenesis of congestive hepatic fibrosis (cardiac cirrhosis)," Hepatology, vol. 21, no. 5, pp. 1232-1237, 1995.

[7] I. Wanless, F. Wong, L. M. Blendis et al., "Hepatic and portal vein thrombosis in cirrhosis: possible role in development of parenchymal extinction and portal hypertension," Hepatology, vol. 21, no. 5, pp. 1238-1247, 1995.

[8] J. W. Chung, G. H. Kim, J. H. Lee et al., "Safety, efficacy, and response predictors of anticoagulation for the treatment of nonmalignant portal-vein thrombosis in patients with cirrhosis: a propensity score matching analysis," Clinical and Molecular Hepatology, vol. 20, no. 4, pp. 384-391, 2014.

[9] E. Villa, C. Cammà, M. Marietta et al., "Enoxaparin prevents portal vein thrombosis and liver decompensation in patients with advanced cirrhosis," Gastroenterology, vol. 143, no. 1253-1260, p. e4, 2012. 
[10] H. Chen, L. Liu, X. Qi et al., "Efficacy and safety of anticoagulation in more advanced portal vein thrombosis in patients with liver cirrhosis," European Journal of Gastroenterology \& Hepatology, vol. 28, no. 1, pp. 82-89, 2016.

[11] L. Loffredo, D. Pastori, A. Farcomeni, and F. Violi, "Effects of anticoagulants in patients with cirrhosis and portal vein thrombosis: a systematic review and meta-analysis," Gastroenterology, vol. 153, no. 2, pp. 480-487, 2017.

[12] K. M. Kassel, B. P. Sullivan, W. Cui, B. L. Copple, and J. P. Luyendyk, "Therapeutic administration of the direct thrombin inhibitor argatroban reduces hepatic inflammation in mice with established fatty liver disease," The American Journal of Pathology, vol. 181, no. 4, pp. 1287-1295, 2012.

[13] W. Li, J. Zhang, Q. Huang, H. Zhu, and X. Zhang, "Long-term administering low anticoagulant activity heparin can lessen rat hepatic fibrosis induced by either $\mathrm{CCl}_{4}$ or porcine serum injection," Hepatology Research, vol. 36, no. 2, pp. 115-123, 2006.

[14] J. Shi, J. H. Hao, W. H. Ren et al., "Effects of heparin on liver fibrosis in patients with chronic hepatitis B," World Journal of Gastroenterology, vol. 9, no. 7, pp. 1611-1614, 2003.

[15] D. J. Tyrrell, A. P. Horne, K. R. Holme, J. M. H. Preuss, and C. P. Page, "Heparin in inflammation: potential therapeutic applications beyond anticoagulation," Advances in Pharmacology, vol. 46, pp. 151-208, 1999.

[16] J.-H. Lee, H. Lee, Y. K. Joung et al., "The use of low molecular weight heparin-pluronic nanogels to impede liver fibrosis by inhibition the TGF- $\beta /$ Smad signaling pathway," Biomaterials, vol. 32, no. 5, pp. 1438-1445, 2011.

[17] M. Vilaseca, H. García-Calderó, E. Lafoz et al., "The anticoagulant rivaroxaban lowers portal hypertension in cirrhotic rats mainly by deactivating hepatic stellate cells," Hepatology, vol. 65, no. 6, pp. 2031-2044, 2017.

[18] N. Bitto, E. Liguori, and V. Mura, "Coagulation, microenvironment and liver fibrosis," Cells, vol. 7, no. 8, p. 85, 2018.

[19] L. Turco, F. Schepis, and E. Villa, "The role of anticoagulation in treating portal hypertension," Current Hepatology Reports, vol. 17, no. 3, pp. 200-208, 2018.

[20] A. Dhar, F. Sadiq, Q. M. Anstee et al., "Thrombin and factor Xa link the coagulation system with liver fibrosis," $B M C$ Gastroenterology, vol. 18, p. 60, 2018.

[21] F. Cerini, M. Vilaseca, E. Lafoz et al., "Enoxaparin reduces hepatic vascular resistance and portal pressure in cirrhotic rats," Journal of Hepatology, vol. 64, no. 4, pp. 834-842, 2016.

[22] J. I. Fortea, A. Zipprich, C. Fernandez-Mena et al., "Enoxaparin does not ameliorate liver fibrosis or portal hypertension in rats with advanced cirrhosis," Liver International, vol. 38, no. 1, pp. 102-112, 2018.

[23] D. Moher, A. Liberati, J. Tetzlaff et al., "Preferred reporting items for systematic reviews and meta-analyses: the PRISMA statement," PLoS Medicine, vol. 6, no. 7, Article ID e1000097, 2009.

[24] Z. D. Goodman, "Grading and staging systems for inflammation and fibrosis in chronic liver diseases," Journal of Hepatology, vol. 47, no. 4, pp. 598-607, 2007.

[25] C. R. Hooijmans, M. M. Rovers, R. B. de Vries et al., "SYRCLE's risk of bias tool for animal studies," BMC Medical Research Methodology, vol. 14, p. 43, 2014.

[26] A. Iorio, F. A. Spencer, M. Falavigna et al., "Use of GRADE for assessment of evidence about prognosis: rating confidence in estimates of event rates in broad categories of patients," $B M J$, vol. 350, no. 7, p. h870, 2015.

[27] S. P. Hozo, B. Djulbegovic, and I. Hozo, "Estimating the mean and variance from the median, range, and the size of a sample," BMC Medical Research Methodology, vol. 20, no. 5, p. 13, 2005.
[28] N. Assy, O. Hussein, A. Khalil et al., "The beneficial effect of aspirin and enoxaparin on fibrosis progression and regenerative activity in a rat model of cirrhosis," Digestive Diseases and Sciences, vol. 52, no. 5, pp. 1187-1193, 2007.

[29] C.-J. Li, Z.-H. Yang, X.-L. Shi, and D.-L. Liu, "Effects of aspirin and enoxaparin in a rat model of liver fibrosis," World Journal of Gastroenterology, vol. 23, no. 35, pp. 6412-6419, 2017.

[30] Y. Yan, C. Guan, S. Du et al., "Effects of enzymatically depolymerized low molecular weight heparins on $\mathrm{CCl}_{4}$-induced liver fibrosis," Frontiers in Pharmacology, vol. 8, p. 514, 2017.

[31] K. Fujita, Y. Nozaki, K. Wada et al., "Effectiveness of antiplatelet drugs against experimental non-alcoholic fatty liver disease," Gut, vol. 57, no. 11, pp. 1583-1591, 2008.

[32] O. M. E. Abdel-Salam, A. R. Baiuomy, A. Ameen, and N. S. Hassan, "A study of unfractionated and low molecular weight heparins in a model of cholestatic liver injury in the rat," Pharmacological Research, vol. 51, no. 1, pp. 59-67, 2005.

[33] W. Abe, K. Ikejima, T. Lang et al., "Low molecular weight heparin prevents hepatic fibrogenesis caused by carbon tetrachloride in the rat," Journal of Hepatology, vol. 46, no. 2, pp. 286-294, 2007.

[34] K.-C. Lee, W.-F. Hsu, Y.-C. Hsieh et al., "Dabigatran reduces liver fibrosis in thioacetamide-injured rats," Digestive Diseases and Sciences, vol. 64, no. 1, pp. 102-112, 2019.

[35] N. I. Mahmoud, B. A. S. Messiha, A. A. Abo-Saif et al., "Inhibition of activated factor X; a new pathway in ameliorating carbon tetrachloride-induced liver fibrosis in rats," Journal of Biochemical and Molecular Toxicology, vol. 33, no. 5, Article ID e22287, 2019.

[36] N. I. Mahmoud, B. A. S. Messiha, I. G. Salehc, A. A. Abo-Saif, and M. S. Abdel-Bakky, "Interruption of platelets and thrombin function as a new approach against liver fibrosis induced experimentally in rats," Life Sciences, vol. 231, Article ID 116522, 2019.

[37] Y. Liu, L. Nong, Y. Jia et al., "Aspirin alleviates hepatic fibrosis by suppressing hepatic stellate cells activation via the TLR4/ NF- $\kappa$ B pathway," Aging, vol. 12, no. 7, pp. 6058-6066, 2020.

[38] M. D. A. Gaça, X. Zhou, and R. C. Benyon, "Regulation of hepatic stellate cell proliferation and collagen synthesis by proteinase-activated receptors," Journal of Hepatology, vol. 36, no. 3, pp. 362-369, 2002.

[39] S. L. Friedman, "Hepatic stellate cells: protean, multifunctional, and enigmatic cells of the liver," Physiological Reviews, vol. 88, no. 1, pp. 125-172, 2008.

[40] W. Ageno, N. Riva, S. Schulman et al., "Long-term clinical outcomes of splanchnic vein thrombosis: results of an international registry," JAMA Internal Medicine, vol. 175, no. 9, pp. 1474-1480, 2015.

[41] G. Bedogni, L. Miglioli, F. Masutti et al., "Natural course of chronic HCV and HBV infection and role of alcohol in the general population: the Dionysos study," The American Journal of Gastroenterology, vol. 103, no. 9, pp. 2248-2253, 2008.

[42] F. Violi, G. R. Corazza, R. G. Corazza et al., "Portal vein thrombosis relevance on liver cirrhosis: Italian venous thrombotic events registry," Internal and Emergency Medicine, vol. 11, no. 8, pp. 1059-1066, 2016.

[43] I. Lurje, L. Hammerich, and F. Tacke, "Dendritic cell and T cell crosstalk in liver fibrogenesis and hepatocarcinogenesis: implications for prevention and therapy of liver cancer," International Journal of Molecular Sciences, vol. 21, no. 19, p. 7378, 2020.

[44] E. Seki and R. F. Schwabe, "Hepatic inflammation and fibrosis: functional links and key pathways," Hepatology, vol. 61, no. 3, pp. 1066-1079, 2015. 
[45] I. Pettinari, R. Vukotic, H. Stefanescu et al., "Clinical impact and safety of anticoagulants for portal vein thrombosis in cirrhosis," The American Journal of Gastroenterology, vol. 114, no. 2, pp. 258-266, 2019.

[46] N. A. Crossley, E. Sena, J. Goehler et al., "Empirical evidence of bias in the design of experimental stroke studies: a metaepidemiologic approach," Stroke, vol. 39, no. 3, pp. 929-934, 2008.

[47] J. A. Hirst, J. Howick, J. K. Aronson et al., "The need for randomization in animal trials: an overview of systematic reviews," PLoS One, vol. 9, no. 6, Article ID e98856, 2014.

[48] E. D. M. Rooke, H. M. Vesterinen, E. S. Sena, K. J. Egan, and M. R. Macleod, "Dopamine agonists in animal models of Parkinson's disease: a systematic review and meta-analysis," Parkinsonism \& Related Disorders, vol. 17, no. 5, pp. 313-320, 2011. 